\title{
Alcohol brief intervention for hospitalized veterans with hazardous drinking: protocol for a 3-arm randomized controlled efficacy trial
}

Lauren M Broyles ${ }^{1,2,3^{*}}$, Melissa E Wieland ${ }^{1}$, Andrea L Confer ${ }^{1}$, Monica M DiNardo ${ }^{1}$, Kevin L Kraemer ${ }^{2}$, Barbara H Hanusa ${ }^{3}$, Ada O Youk ${ }^{1,4}$, Adam J Gordon ${ }^{1,2,3}$ and Mary Ann Sevick ${ }^{5}$

\begin{abstract}
Background: Various hospital accreditation and quality assurance entities in the United States have approved and endorsed performance measures promoting alcohol brief intervention (BI) for hospitalized individuals who screen positive for unhealthy alcohol use, the spectrum of use ranging from hazardous use to alcohol use disorders. These performance measures have been controversial due to the limited and equivocal evidence for the efficacy of BI among hospitalized individuals. The few BI trials conducted with hospital inpatients vary widely in methodological quality. While the majority of these studies indicate limited to no effects of BI in this population, none have been designed to account for the most pervasive methodological issue in Bl studies presumed to drive study findings towards the null: assessment reactivity (AR).
\end{abstract}

Methods/Design: This is a three-arm, single-site, randomized controlled trial of BI for hospitalized patients at a large academic medical center affiliated with the U.S. Department of Veterans Affairs who use alcohol at hazardous levels but do not have an alcohol use disorder. Participants are randomized to one of three study conditions. Study Arm 1 receives a three-part alcohol Bl. Study Arm 2 receives attention control. To account for potential AR, Study Arm 3 receives $A C$ with limited assessment. Primary outcomes will include the number of standard drinks/week and binge drinking episodes reported in the 30-day period prior to a final measurement visit obtained 6 months after hospital discharge. Additional outcomes will include readiness to change drinking behavior and number of adverse consequences of alcohol use. To assess differences in primary outcomes across the three arms, we will use mixed-effects regression models that account for a patient's repeated measures over the timepoints and clustering within medical units. Intervention implementation will be assessed by: a) review of intervention audio recordings to characterize barriers to intervention fidelity; and b) feasibility of participant recruitment, enrollment, and follow-up.

Discussion: The results of this methodologically rigorous trial will provide greater justification for or against the use of $\mathrm{Bl}$ performance measures in the inpatient setting and inform organizational responses to Bl-related hospital accreditation and performance measures.

Trial registration: NCT01602172

Keywords: Inpatients, Risk reduction behavior, Drinking behavior, Alcohol drinking, Binge drinking, Brief intervention, Clinical trial, Assessment reactivity, Measurement effects

\footnotetext{
* Correspondence: lauren.broyles@va.gov

${ }^{1}$ Center for Health Equity Research and Promotion, Veterans Affairs (VA)

Pittsburgh Healthcare System, University Drive C (151C), Pittsburgh, PA

15240, USA

${ }^{2}$ Division of General Internal Medicine, Department of Medicine, School of

Medicine, University of Pittsburgh, 230 McKee Place, Suite 600, Pittsburgh, PA

15213, USA

Full list of author information is available at the end of the article
}

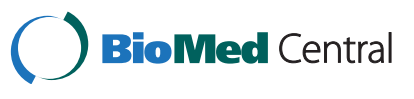

(C) 2015 Broyles et al.; licensee BioMed Central. This is an Open Access article distributed under the terms of the Creative Commons Attribution License (http://creativecommons.org/licenses/by/4.0), which permits unrestricted use, distribution, and reproduction in any medium, provided the original work is properly credited. The Creative Commons Public Domain Dedication waiver (http://creativecommons.org/publicdomain/zero/1.0/) applies to the data made available in this article, unless otherwise stated. 


\section{Background}

Unhealthy alcohol use comprises the spectrum of alcohol use, ranging from hazardous drinking, (defined as consumption that exceeds 14 standard drinks per week or 4 per occasion for men, and 7 standard drinks per week or 3 per occasion for women and healthy individuals age 65 or older) to alcohol use disorders [1-3]. In 2008, The Joint Commission (TJC), a hospital accreditation body in the United States, began the development and testing of a set of standardized hospital performance measures to address the entire spectrum of alcohol use through screening, brief intervention (BI) counseling, referral to specialty treatment, pharmacotherapy, and follow-up for hospitalized patients [4]. Because the measures are designed to be applicable to all hospitalized patients, regardless of reason for admission, they are categorized as global performance measures. The measures are not mandatory, but in January 2012, they began being offered as one of the sets that hospitals could select to achieve in order to receive ongoing accreditation [5]. In February 2014, the measures were endorsed by the U.S. National Quality Forum [6]. However, during their initial development and release, the measures generated considerable debate [7-11]. Opponents of the measures expressed concerns regarding the limited and equivocal nature of the evidence for efficacy of BI among hospitalized individuals. [7,8,12,13]. Meanwhile, proponents of the measures endorsed their uptake and implementation, challenging the need for continued alcohol BI trials across different health-care settings for patients with varying levels of alcohol involvement and for different combinations of substances [11].

At the time of this debate, few trials of alcohol BI had even been conducted in hospitalized patients [12,14-16], with only one of these conducted in a sample of U.S. drinkers [12]. Because cultural norms and attitudes about alcohol use vary internationally, caution must be exercised in generalizing the results of BI trials from outside the United States. Additionally, while the methodological quality of these BI studies varies widely, the majority did not demonstrate significant reductions in alcohol consumption or in the adverse consequences of alcohol use $[12,14,16]$. Subsequent post-hoc analyses, however, suggested that BI was in fact efficacious for decreasing alcohol consumption at 3 months among nondependent, hazardous drinkers (i.e., patients whose use met criteria for hazardous drinking but did not simultaneously meet criteria for alcohol dependence) [13]. Additionally, patients consuming alcohol at nondependent, hazardous levels who had an alcohol-attributable admitting diagnosis had significantly fewer heavy episodic (binge) drinking days at 3-month follow-up [17].

Since this active debate, three additional trials of BI for hospitalized patients have been conducted outside the
United States, but again, with equivocal results and/or coarser outcomes, such as change in alcohol screening status, as opposed to more specific measures of alcohol consumption [18-20]. BI researchers have long suggested that null or inconsistent findings in their trials are attributable to the fact that control groups in these studies regularly showed decreased alcohol consumption, thus reducing the ability to demonstrate BI's effects [21,22]. Furthermore, these reductions in control group drinking have consistently been explained by assessment reactivity (AR) - the potential for extensive and/or repeated study assessments of alcohol use to mimic the components of alcohol BI. When AR occurs, study results can be biased towards the null because in essence, both study groups inadvertently receive some degree of intervention [21-23]. Despite the frequency with which BI investigators ascribe null findings to $A R$, few researchers have designed BI trials to take its potential effects into account [21,24-26], and none have sought to do so in trials of hospitalized individuals.

Our overarching goal is to inform the debate about the efficacy of alcohol BI for hospitalized individuals in a methodologically rigorous trial using a U.S.-based sample of hazardous drinkers. Furthermore, our study design addresses several key methodological issues that have been raised as concerns regarding other BI trials. To address (or avoid) AR, we employ a threearm randomized design: Arm 1 consists of veterans randomized to $\mathrm{BI}$ (BI); Arm 2 consists of veterans randomized to Attention Control (AC); and Arm 3 consists of veterans randomized to an $\mathrm{AC}$ group with limited assessment (AC-LA) (i.e., alcohol consumption variables only).

\section{Specific aims and hypotheses}

The Specific Aims of this three-arm randomized controlled trial (RCT) are to: (1) determine the impact of BI on the alcohol screening status, number of drinks/week, number of binge drinking episodes, readiness to change drinking behavior, and adverse consequences of alcohol use in hospitalized hazardous drinkers; and (2) evaluate the process of intervention implementation by characterizing barriers to intervention fidelity and assessing the feasibility of recruitment, enrollment, and follow-up of hospitalized hazardous drinkers.

We hypothesize that at 6 months post-discharge, Arm 1 (BI) will report greater reductions in the alcohol consumption outcomes than Arms 2 (AC) and 3 (AC-LA). Furthermore, we hypothesize that AR will result in some reductions in alcohol consumption in Arm 2, and that the reductions in Arm 2 will be greater than those displayed in Arm 3, but less than those displayed by Arm 1. 


\section{Methods}

\section{Human subjects protections}

This study is approved by the Research and Development Service and the Institutional Review Board (IRB) of the U.S. Department of Veterans Affairs (VA) Pittsburgh Healthcare System (VAPHS).

\section{Design}

This study is a three-arm, single-site, RCT of BI for hospitalized, hazardous drinkers. Participants are randomized to one of three study conditions: Arm 1: a group receiving a 3-part alcohol BI; Arm 2: an AC group; and Arm 3: an AC-LA group.

\section{Setting and participants}

This study is being conducted on the three medicalsurgical (Med-Surg) units at the University Drive campus of the VAPHS, a large academic medical center in southwestern Pennsylvania that is part of the VA. The University Drive campus is home to a 146-bed hospital facility that provides routine medical, neurological, psychiatric, and surgical care in addition to specialized services. Patient and unit characteristics for the three participating Med-Surg units are provided in Table 1.

Patient inclusion criteria for the study are: admission to a participating unit; age 21 or older; and hazardous, nondependent alcohol consumption (defined in Screening section below). Study exclusion criteria are: participation in alcohol treatment and/or 12-step programs in the previous 6 months; current drug or alcohol dependence; significant cognitive impairment; diagnosis of a bipolar or psychotic disorder; residence in a restricted housing setting (e.g., skilled nursing facility); lack of telephone access or unwillingness to be contacted for follow-up; sensory impairment precluding communication; and medically-related inability to participate or consent to study participation.

Table 1 Medical-surgical unit and patient profile, VAPHS, FY 2010

\begin{tabular}{|c|c|c|c|c|}
\hline \multicolumn{5}{|c|}{ Parameter type of unit total for 3 units } \\
\hline & & Medical & Surgical & Total \\
\hline Admissions/month & & 407 & 240 & 647 \\
\hline Average daily census & & 67 & 49 & 58 \\
\hline \multirow[t]{3}{*}{ Average length of stay (days) } & Unit A & Unit B & Unit C & \\
\hline & (Surg) & (Med-Tele) & (Med-Liver) & 3.9 \\
\hline & 3.6 & 3.1 & 5.0 & \\
\hline Female veterans (\%) & & & & 4 \\
\hline OEF/OIF veterans (\%) & & & & 2 \\
\hline Average patient age & & & & 65 \\
\hline$\%$ Age 50 or under & & & & 10 \\
\hline
\end{tabular}

Note: Med = Medical; Liver = Liver Transplant; Surg = Surgical; Tele = Telemetry; VAPHS = VA Pittsburgh Healthcare System.

\section{Recruitment, screening, and enrollment}

In the inpatient care setting, the logistics of screening and recruitment of hospitalized patients for research study participation require careful consideration. Adherence to federal and local human subjects protections for research can be challenged by patient acuity, inpatient processes of care (e.g., medication administration/side effects, bedside and off-unit procedures), and the need for countless assessments by professionals from multiple disciplines and sub-specialties. Our multi-step procedures for prescreening, approach, screening, informed consent, and study enrollment are described below and depicted in Figure 1.

\section{Prescreening}

Because of the high volume of admissions to the participating units (approximately 650 admissions each month), we have a Waiver of Informed Consent from the IRB at VAPHS that authorizes us to generate a daily list of patients admitted to the participating units and allows for review of each admitted patient's electronic medical record (EMR) in order to identify ineligibility criteria (e.g., housing status, psychiatric diagnosis). This prescreening process allows us to avoid exerting unnecessary burden on acutely ill patients who could be determined ineligible by record review, and reduces the volume of patients to be approached for interest and screening. After review of the EMR, a list of patients who remain eligible for the study is prepared for each unit.

\section{Screening}

According to VAPHS IRB regulations, patients may not be directly approached about research participation by individuals who are not directly associated with the patient's care; a clinical care provider must first secure permission for a member of the research staff to approach the patient. For this reason, our study's Research Assistant (RA) first visits each of the three participating units and presents the charge nurse with the list of the prescreened patients admitted to that unit. The charge nurse (or delegate) then approaches each patient on the list to request his/her permission to allow the RA to approach the patient (i.e., enter the room to provide a study overview and begin the recruitment process). The RA then provides the patient with a general description of the study. If interested, the patient is asked to provide verbal consent for eligibility screening, which is performed by the RA. In Table 2, the four-step study screening process is represented by the "Eligibility Screening (ES)" column for each study arm.

Because hospitalized individuals may have altered mental status due to acute illness or receipt of sedating medication, the Short Blessed Test (SBT) is first performed to assess for cognitive impairment. The SBT is a 


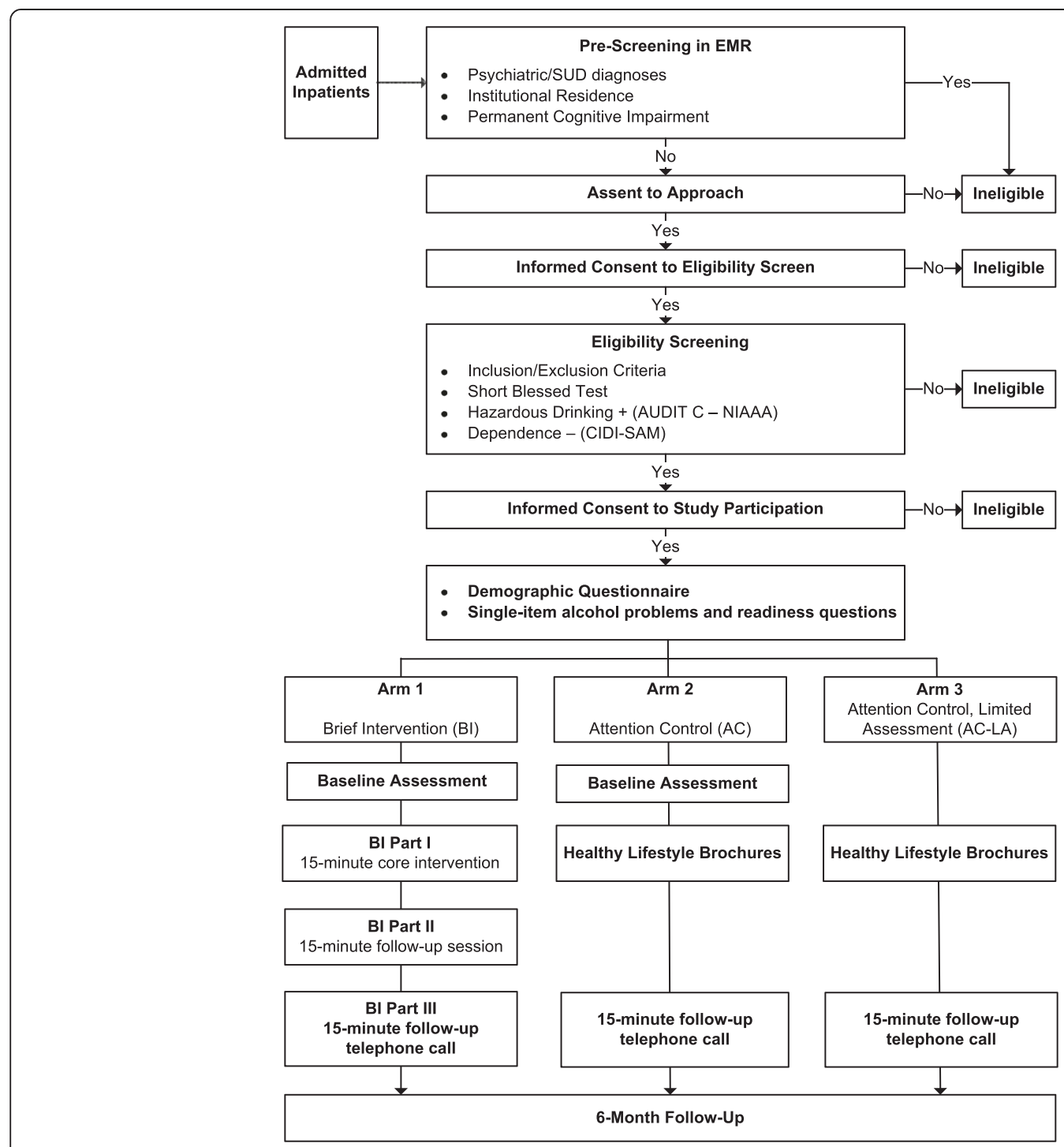

Figure 1 Study design and procedures.

6-item screening instrument used to identify cognitive deficits in orientation, registration, and attention [27]. Patients are then assessed for hazardous alcohol consumption using criteria from the U.S. National Institute on Alcohol Abuse and Alcoholism (NIAAA). Individuals who consume more than 14 drinks/week or 4 drinks/occasion (men); or more than 7 drinks/week or 3 drinks/ occasion (women) are considered hazardous drinkers [2]. Number of drinks consumed per week is determined by the product of responses to the following two questions: (1) On average, how many days a week do you have an alcoholic drink? and (2) On a typical drinking day, how many standard-sized drinks do you have? [28]. A "standard-sized drink" refers to 12 ounces of beer, 5 ounces of wine, or 1.5 ounces of liquor/spirits [2]. Number of binge drinking episodes is assessed through a third question: How many times in the past 30 days have you had 5 or more standard-sized drinks in a day (men), or 4 or more standard-sized drinks in a day? (women) [2]. The use of NIAAA criteria to determine the need for an alcohol BI provides us with precise quantification of alcohol use for measurement of our primary outcome (number of drinks/week) and is consistent with VA/Department of Defense Clinical Practice Guidelines, which recommend BI for individuals who drink above the specified limits or who drink despite contraindications [28].

Potential participants also complete the Alcohol Use Disorders Identification Test - Consumption (AUDIT-C) to provide an additional alcohol outcome measure that is consistent with and easily comparable to clinical practice in VA primary care and TJC measure specifications. The AUDIT-C is a widely used and validated three-item alcohol screen that can help identify persons who are hazardous drinkers. The AUDIT-C is scored on a scale of $0-12$. For 
Table 2 Study assessments by study arm and timepoint

\begin{tabular}{|c|c|c|c|c|c|c|c|c|c|c|c|c|c|}
\hline \multirow[b]{3}{*}{ Variable } & \multirow[b]{3}{*}{ Instrument } & \multirow{2}{*}{\multicolumn{4}{|c|}{$\begin{array}{l}\text { Arm } 1 \text { brief } \\
\text { intervention (BI) }\end{array}$}} & \multirow{2}{*}{\multicolumn{4}{|c|}{$\begin{array}{l}\text { Arm } 2 \text { attention } \\
\text { control (AC) } \\
\text { Timepoint }\end{array}$}} & \multirow{2}{*}{\multicolumn{4}{|c|}{$\begin{array}{l}\text { Arm } 3 \text { attention } \\
\text { control, limited } \\
\text { assessment (AC-LA) } \\
\text { Timepoint }\end{array}$}} \\
\hline & & & & & & & & & & & & & \\
\hline & & ES & PC & B & FU & ES & PC & B & FU & ES & PC & B & FU \\
\hline Cognitive status & Short Blessed Test & $x$ & & & & $x$ & & & & $x$ & & & \\
\hline Alcohol screening status & AUDIT-C & $x$ & & & $x$ & $x$ & & & $x$ & $x$ & & & $x$ \\
\hline Drinks per week and Binge episodes last 30 days & 3 NIAAA questions & $x$ & & & $x$ & $x$ & & & $x$ & $x$ & & & $x$ \\
\hline Alcohol dependence & Modified CIDI-SAM & $x$ & & & & $x$ & & & & $x$ & & & \\
\hline Sociodemographic/ clinical data & Sociodemographic form & & $x$ & & $x$ & & $x$ & & $x$ & & $x$ & & $x$ \\
\hline $\begin{array}{l}\text { Readiness to change drinking behavior and } \\
\text { adverse consequences of alcohol use }\end{array}$ & $\begin{array}{l}\text { Single items from SOCRATES } \\
\text { and SIP-2R }\end{array}$ & & & & & & & & & & $x$ & & \\
\hline Readiness to change drinking behavior & SOCRATES & & & $x$ & $x$ & & & $x$ & $x$ & & & & $x$ \\
\hline Adverse consequences of alcohol use & SIP-2R & & & $x$ & $x$ & & & $x$ & $x$ & & & & $x$ \\
\hline $\begin{array}{l}\text { Participant responsiveness (patient opinions } \\
\text { about the intervention) }\end{array}$ & $\begin{array}{l}\text { 4-item investigator developed } \\
\text { instrument }\end{array}$ & & & $x$ & & & & & & & & & \\
\hline
\end{tabular}

Note: AUDIT-C = Alcohol Use Disorders Identification Test, Consumption; NIAAA = National Institute on Alcohol Abuse and Alcoholism; SOCRATES = Stages of Change Readiness and Treatment Eagerness Scale; SIP-2R = Short Inventory of Problems; Timepoints $-E S=$ Eligibility Screening (10-25 minutes); PC = Post Consent (5 minutes); $\mathrm{B}=$ Baseline (10 minutes); $\mathrm{FU}=$ Follow-up at 6 months (30-45 minutes).

men, total scores of 4 or more are considered positive; for women, scores of 3 or more are considered positive [29,30].

Section C (Alcohol) from the Composite International Diagnostic Interview, Substance Abuse Module (CIDISAM) [31] is administered to rule out hazardous drinkers who also meet criteria for alcohol dependence (Table 2). The CIDI-SAM is a comprehensive, fully structured diagnostic interview for the assessment of mental disorders that provides, by means of algorithms, lifetime and current diagnoses according to the accepted definitions of ICD-10 and the Diagnostic and Statistical Manual of Mental Disorders, Fourth Edition (DSM-IV) [31,32]. Section C of the CIDI-SAM can be administered by trained clinician or nonclinician interviewers in approximately 15 minutes in general populations outside of psychiatric treatment settings [31]. In partnership with the instrument developers, we modified Section $C$ of the CIDI-SAM to facilitate its use in the specific context of this research study (for the rapid identification of potential alcohol dependence to determine study eligibility). All staff were trained by the instrument developers to administer this modified instrument, which contained items C16-C18, C20, C25-C30, C33, and CARD 7 of the original CIDI-SAM.

Hazardous drinkers who do not meet criteria for alcohol dependence according to CIDI-SAM are eligible for study participation. Eligible individuals then complete the informed consent process with the RA. While the RA then determines the patient's randomization assignment, each patient completes a basic investigator-developed sociodemographic and clinical information form. General health behavior questions extracted directly from the Behavioral Risk Factor Surveillance System questionnaire are embedded within the sociodemographic form to assess other health behaviors such as tobacco use, physical activity, and diet [31,33].

\section{Randomization}

Participants are randomized to one of the three study conditions (arms) described below. Randomization assignments were generated by the study statistician prior to study start and placed in masked envelopes. Randomization sequences were stratified by sex, race, and hospital unit in a $2: 1: 1$ ratio. This unequal allocation to the three study conditions allows more veterans to be exposed to the expected efficacious intervention and more observations to be available for analyses of mediators and moderators of positive outcomes in the BI group.

\section{Arm 1: Brief intervention}

Arm 1 comprises the experimental BI condition (Table 3). The three-part BI is adapted from the Brief Negotiated Interview [34] and supplemented with alcohol-related patient education materials available through the VA $[35,36]$ and NIAAA [37]. Part I, the core BI, consists of a multicomponent motivational discussion with the nurse interventionist within 12 hours of baseline assessment, which includes personalized risk feedback, advice to abstain or reduce consumption, and the negotiation of an individual change plan $[28,34,38,39]$. Part II consists of a single, brief, in-hospital or telephone follow-up session, with the same nurse interventionist, to briefly reinforce the original intervention, discuss and "troubleshoot" the patient's change plan, and offer additional encouragement. Part III of the intervention consists of a brief follow-up telephone call 2 weeks following hospital discharge, during which the 
Table 3 Components of 3-part brief intervention for hospitalized hazardous drinkers
Part I: Core brief intervention ( 15 minutes) [28,34,38,39]
- Expression of concern about the patient's level of alcohol consumption
- Personalized feedback linking alcohol use and health
- Linkage of alcohol to admitting diagnosis, if applicable
- Advice to abstain/reduce alcohol consumption to below NIAAA limits
- Assessment of motivation/readiness to change drinking behavior
- Support for choosing an alcohol consumption goal
- Strategies/encouragement for reaching alcohol consumption goal (change plan)
- Potential referral to specialty care or additional support

Part II: In-hospital follow-up session (12-24 hours after core brief intervention, $\sim 15$ minutes)

- Basic reinforcement of core brief intervention

- Re-assessment and support of motivation and review of change plan (if applicable)

- Discussion of challenges/barriers to change

- Consideration of additional strategies for change plan

- Support and encouragement for reaching goal

Part III: Telephone follow-up session ( 2 weeks post-initial brief intervention, $\sim 15$ minutes)

- Same as part II

same nurse interventionist again briefly reinforces the original intervention, discusses the patient's change plan, and offers additional encouragement (Table 3). Typical BI follow-up periods for hospitalized patients range from one week (for written materials) to one month (for additional intervention) [12,40]. We selected a 2-week period in order to verbally reinforce recent intervention material, while allowing recently hospitalized patients time to readjust and resume self-care practices. For patients who do not initially establish an alcohol consumption goal or initiate a change plan during the initial $\mathrm{BI}$, the followup session focuses on exploring and supporting general readiness to change.

\section{Arms 2 \& 3: Control conditions}

Arm 2 (AC) and Arm 3 (AC-LA) comprise the control conditions. Patients in Arms 2 and 3 receive healthy lifestyle brochures that address general healthy lifestyle behaviors such as dietary recommendations, tobacco cessation, and weight management. Brochures were deliberately selected that do not contain content similar to that of the alcohol BI. To ensure parallel attention and number of contacts across groups [41], patients in Arms 2 and 3 also receive a 5-15 minute follow-up telephone call at 2 weeks post-discharge to inquire about general well-being and to review brochure content. Arms
2 and 3 serve as "usual care" insofar as alcohol screening or intervention for hazardous drinking is not routine standard of care for hospitalized patients at this VA medical center.

\section{Baseline assessment}

The extent of patients' baseline assessments depends on their randomization assignment (Table 2). As previously mentioned, all alcohol consumption data related to the primary outcomes are gathered by the RA during eligibility screening. All sociodemographic/clinical data are gathered immediately prior to randomization. Participants in Arms 1 and 2 are administered the Stages of Change Readiness and Treatment Eagerness Scale (SOCRATES) and the Short Inventory of Problems (SIP-2R) instruments by the RA. The SOCRATES, Version 8 , is a 19-item instrument with three subscales: Recognition (of a potential alcohol problem), Ambivalence (about one's drinking), and Taking Steps (towards behavior change) [42]. Normative data are available to aid in subscale score interpretation. We assess adverse consequences of alcohol use with the SIP-2R, a widely used 15-item, standalone, short version of the Drinker Inventory of Consequences scale [43]. This instrument assesses adverse consequences of alcohol use over the past 3 months in five areas: interpersonal, physical, social, impulsive, and intrapersonal [43]. The SOCRATES and SIP-2R instruments are not administered to Arm 3 participants to reduce the possible impact of AR. Patients randomized to the Arm 3 condition receive only two single items from the SOCRATES and SIP-2R questionnaires that are embedded within the demographic/ clinical questionnaire for all participants; this allows for a coarse measure of these constructs in Arm 3 patients.

\section{Follow-up assessment at 6 months}

At 6 months post-discharge, all participants receive the following assessments from the RA by telephone: the sociodemographic questionnaire with embedded general health questions; the AUDIT-C; NIAAA questions regarding the number of drinks consumed per week and number of binge drinking episodes in the past 30 days; the SOCRATES; and the SIP-2R (see follow-up column, Table 2).

\section{Assessment of intervention fidelity (Specific aim 2)}

We are assessing the five classic components of intervention fidelity: quality of delivery, exposure, adherence, participant responsiveness, and program differentiation [44]. These components are defined as follows: (1) quality of delivery: the extent to which all six FRAMES counseling components (feedback, responsibility, advice to change, menu of options, empathy, self-efficacy) [45] and three specific motivational interviewing (MI) techniques (reflective listening, decisional balance, assessment 
of readiness) [45] are included in the intervention; (2) exposure: the duration of the intervention (minutes); (3) adherence: the number and character of procedural deviations and interruptions to intervention delivery; (4) participant responsiveness: patient opinions about the intervention; and (5) program differentiation: the extent to which only the BI group receives alcoholrelated feedback and counseling (i.e., avoiding "crosscontamination" across conditions).

Intervention fidelity is assessed for Parts I and II of the intervention using several data collection methods. First, we are audio recording 100 percent of the Part I interventions. The Study Coordinator will review 50 percent of the audio recordings (randomly selected and stratified by unit over time) to assess quality of delivery, exposure, and adherence. Barriers to intervention fidelity are assessed using several data collection methods. First, the nurse interventionist maintains narrative field notes on each intervention session, focusing on facilitators and barriers to intervention delivery and patient response to the intervention. Quality of delivery is rated using modified versions of established checklists for $\mathrm{BI}$, which are based on the FRAMES model and principles of MI [45-47], upon which BI is based. For the recorded interventions and follow-up calls, we compare the interventionist's notes to the content of the phone calls. Participant responsiveness is assessed at the conclusion of each intervention. Participants indicate their level of agreement with four statements addressing perceptions of the intervention (e.g., helpfulness, supportiveness) using a 7-point Likert-style scale, and briefly explain each answer in a free-text format below each item. Program differentiation is assessed by asking all groups at the 2-week follow-up call and the 6-month timepoint to recall the extent to which they received any additional alcohol-related feedback, recommendations, or counseling (e.g., primary care provider). These questions are embedded in the sociodemographic/clinical form.

\section{Nurse interventionist training}

Prior to the study, the nurse interventionist, a member of the research team, received a 2-day intensive MI training led by certified trainers from the Motivational Interviewing Network of Trainers [48]. The Principal Investigator provided additional training over the course of one month that was specific to the use of BI techniques. This training included additional role-plays featuring inpatient care scenarios with staff serving as "standardized patients", as well as training DVDs and webinars demonstrating effective BI patient interactions.

\section{Analysis plan}

For Specific Aim 1, data analysis will focus on the change in alcohol consumption variables from baseline to 6 months across the three arms and, to the extent possible, exploratory analyses of moderators and mediators of the observed effects. The primary independent variable is the randomized treatment group. The primary outcomes of interest are: alcohol screening status, number of drinks/week (past 30 days), and number of binge drinking episodes (past 30 days). Additional outcomes include readiness to change drinking behavior and number of adverse consequences of alcohol use (for patients in Arms 1 and 2 only). Specific Aim 1 will be tested with mixed-effects regression models that account for withinpatient correlation and clustering of patients within medical units. To further elucidate the impact of BI on the outcomes of interest, additional post-hoc tests for all pair-wise comparisons (i.e., BI vs. AC, BI vs. AC-LA, AC vs. AC-LA) will be conducted.

To the extent possible, we will also conduct exploratory analyses of potential moderators and mediators of effects due to the intervention. Comparisons between Arm 1 and Arm 2 can include more variables related to the underlying positive changes that $\mathrm{BI}$ is designed to facilitate. In particular, we will be able to explore whether the baseline SOCRATES and SIP are important moderators of change in drinking behaviors, in addition to the demographic moderators.

A sample size of 320 that complete the study (160 BI, 80 Attention Control, 80 Attention Control, Limited Assessment) will allow us to detect mean differences of 2.6 drinks per week and 0.34 points on the AUDIT-C. Sample size estimates included expected accrual of veteran inpatients into the study, duration of study participation (6 months), and number of patients expected to be lost to follow-up based on previous BI studies of hospitalized patients $(20-30 \%)[12,14,16,40]$.

To characterize barriers to intervention fidelity (Specific Aim 2), we will use basic descriptive statistics (e.g., frequency counts, means, medians) to describe the numbers of deviations and interruptions to intervention delivery as planned. Based on the interventionist field notes, we will categorize the types of deviations/interruptions. We will also use these descriptive statistics to describe duration of the intervention (in minutes), the presence/absence of each FRAMES and MI technique within the intervention, and the receipt/nonreceipt of additional alcohol feedback, advice, or counseling. We will also categorize the types of additional feedback/counseling received, the provider involved, and the context in which it occurred. Participant responsiveness data (opinions about the intervention) will be analyzed with frequency counts and simple content analysis of free-text comments.

Additionally, to characterize eligibility, recruitment, and retention of participants, we will develop a recruitment funnel to assess: (a) rates of patient eligibility among hospitalized veterans; (b) rates of refusal for screening; and (c) rates of refusal for study enrollment. 
We will determine the: (d) mean number of telephone attempts to contact participants at 2 weeks and 6 months; and the (e) proportion of successful to unsuccessful telephone contacts at 2 weeks and 6 months. We will also categorize and generate frequency counts of reasons for: (f) patient ineligibility; (g) screening refusal, and (h) enrollment refusal.

\section{Discussion}

Hospitalization can be a window of opportunity in which to engage the patient who is drinking at hazardous levels in a BI discussion, linking alcohol use to acute/chronic health problems, and then assessing and supporting personal motivation to change drinking behavior, particularly if the hospital admission is alcohol related. This preventative, public health-oriented approach is designed to help avert the physical and psychosocial consequences of hazardous alcohol consumption for individuals and their families. Based on this rationale, and on evidence of the efficacy of $\mathrm{BI}$ in other health-care settings, hospital accreditation and quality assurance bodies in the United States have issued and endorsed performance measures pertaining to alcohol BI for hospitalized patients.

Several features of our trial represent improvements in study design over other BI trials, particularly those with inpatients; these features are discussed in the following sections.

\section{Attention to potential AR}

Our three-arm design allows us to compare the effect of the intervention with that of usual care in the presence or absence of extensive alcohol-related assessment. Unequal changes in the primary dependent variables (number of drinks/week, number of binge drinking episodes, change in alcohol screening status) across the two control groups will suggest the presence of AR. To further reduce potential $\mathrm{AR}$, we are limiting the number, scope, and frequency of assessments [21]. We are using only one instrument each for two of the five dependent variables and are limiting the assessment of alcohol use/hazardous drinking to six items. Also, to minimize AR, we have only one assessment timepoint following baseline. Additionally, normative feedback about drinking behavior to both control group participants is provided within the context of general health maintenance recommendations [21].

\section{Assessment of intervention fidelity}

Assessment of intervention fidelity focuses on factors that impede delivery of the intervention and avoid interventionist drift. In our prior work, inpatient nurses identified numerous factors that could affect intervention fidelity and feasibility (e.g., time, potential interruptions for clinical care, and patient privacy and engagement) [49]. Features of acute care delivery and the inpatient setting, such as frequent need for clinical care/testing and shared patient rooms, may significantly impede BI delivery and compromise intervention integrity [50]. Only one other BI study among hospitalized patients has reported efforts to ensure intervention fidelity [12]. Other negative trials either explicitly report difficulties ensuring intervention fidelity (e.g., intervention duration, techniques) [14], or do not report on intervention fidelity at all $[15,16]$.

\section{Limitation of the entire sample to hazardous, nondependent drinkers}

Hazardous, nondependent drinkers are the population for whom BI was intended and in whom it has the greatest demonstrated efficacy $[51,52]$. Individuals with alcohol dependence typically require treatment of greater intensity and duration than BI provides, and particular attention to issues of loss of control, tolerance, and withdrawal. Other (null) trials of BI for hospitalized patients have included heavier-drinking and/or dependent individuals $[12,14,19]$. However, as noted earlier, in one case, when the sample was later restricted to hazardous, nondependent users, effects for reduced alcohol consumption were in fact detected [13]. We consider inclusion of only hazardous drinkers in the sample to be a strength because it eliminates the potential "noise" (and null effects) caused by including individuals in the sample for whom BI is not intended.

This study is not designed to answer questions of BI's efficacy in other groups of drinkers (e.g., dependent individuals), where evidence for the efficacy of $\mathrm{BI}$ is uncertain $[51,53]$. Nonetheless, we recognize the fact that TJC measures do require BI for all patients with a positive alcohol screening (thus potentially requiring BI for patients who also meet criteria for alcohol dependence). Our study results will not be able to address the efficacy of $\mathrm{BI}$ for individuals with an alcohol use disorder.

\section{Patient-level randomization and unequal allocation of patients to groups in a 2:1:1 ratio}

This aspect of study design allows more veterans to be exposed to the expected efficacious intervention and permits a more economically feasible study, because fewer hospital units will be needed than with cluster randomization. Also, each hospital unit has its own unique staff, administration, and culture that could influence the delivery and efficacy of the BI. With our design, unit characteristics are equally distributed across participants.

\section{A three-part BI discussion that includes two follow-up sessions}

Follow-up sessions have been recommended by other researchers to increase the effect and salience of the BI 
$[12,40]$ and to allow exploration of ways to integrate inpatient BI with ongoing attention to hazardous alcohol use in primary care, post-discharge.

\section{Additional considerations}

As our overarching goal is to inform the debate about the efficacy of alcohol BI for hospitalized individuals in the context of imminent widespread adoption of TJC hospital performance measures, this study was not designed as an effectiveness trial that mirrors the measures' current "real-life" specifications, particularly since such measures are subject to modification by TJC as well as VA's own External Peer Review Program. Nonetheless, we recognize that our use of the NIAAA questions to determine study eligibility/need for a BI is slightly different from what is specified in the measures from TJC. Specifically, TJC measures dictate that individuals be screened using a validated screening instrument (such as the AUDIT-C) and that individuals with a positive alcohol screen must then receive a BI [4]. Additional assessment of alcohol consumption is not required to make the determination of need for a BI. In contrast, in the VA Substance Use Disorder Clinical Practice Guidelines, the need for BI is not determined by screening result, but by the results of "additional assessment" (i.e., determination of whether the patient is drinking above the NIAAA limits or drinking despite contraindications) [28].

We nonetheless anticipate that the applicability of our results from this trial will not be severely hampered by these differences in use of the NIAAA questions versus AUDIT-C to determine need for BI. If our study ultimately demonstrates the efficacy of BI for reducing drinking in hospitalized patients, then one could argue that we have generated evidence for the efficacy of BI in a "more severe" patient population of drinkers (i.e., those who exceed the NIAAA limits) and that its efficacy in a "less severe" group (i.e., those who "only" had a positive AUDIT-C screen) would still be in question. However, this assertion would be valid only if the AUDIT-C has a high rate of false negatives (i.e., patients who score negative on the AUDIT-C despite drinking above the NIAAA-specified limits). Importantly, in one recent study of almost 500,000 individuals, only about five percent of men and three percent of women in the study had negative AUDIT-C screens, despite reported heavy episodic drinking (i.e., drinking above daily limits) [54].

\section{Conclusion}

The results of this trial will provide greater justification for or against the use of BI performance measures in the inpatient setting, as well as help inform organizational responses to BI-related hospital accreditation and performance measures from TJC and other entities interested in quality of care. Our intervention fidelity and execution data can also inform the specifications of such measures and their implementation. Administrators and clinicians charged with alcohol-related performance measurement and corresponding BI implementation will require efficacious, practical, and patient-centered BI delivery models.

\section{Abbreviations \\ AC: Attention control; AC-LA: Attention control - limited assessment; AR: Assessment reactivity; AUDIT-C: Alcohol use disorders identification test - consumption; BI: Brief intervention; CIDI-SAM: Composite international diagnostic interview, substance abuse module; DSM: Diagnostic and statistical manual of mental disorders; EMR: Electronic medical record; ES: Eligibility screening; FRAMES: Feedback, responsibility, advice to change, menu of options, empathy, self-efficacy; IRB: Institutional review board; MI: Motivational interviewing; MINT: Motivational interviewing network of trainers; NIAAA: National institute on alcohol abuse and alcoholism; OEF: Operation enduring freedom; OIF: Operation Iraqi freedom; PI: Principal investigator; RA: Research assistant; SIP-2R: Short inventory of problems - short version; SOCRATES: Stages of change readiness and treatment eagerness scale; TJC: The joint commission; VA: Veterans affairs; VAPHS: VA Pittsburgh healthcare system.}

\section{Competing interests}

The authors declare that they have no competing interests.

\section{Authors' contributions}

$L M B, M A S, A J G$, and KLK conceptualized the study and obtained funding. MEW has overall responsibility for the execution and coordination of the study, overseeing data collection, intervention fidelity, and human subjects protections. MEW also contributed substantially to the first draft of the manuscript. ALC serves as the patient recruiter, and has primary responsibility for data collection and management. She performed literature searches and reviews and contributed to the first draft of the manuscript. MMD serves as the nurse interventionist, having primary responsibility for delivery of the three-part alcohol BI. She also assists with data management. BHH developed the initial analytic plan for the study. AOY is the senior statistician overseeing and conducting quantitative data analysis. All authors read and approved the final manuscript.

\section{Authors' information}

LMB is a Research Health Scientist at the Center for Health Equity Research and Promotion (CHERP), and the VISN 4 Mental Illness Research, Education, and Clinical Center (MIRECC) at VAPHS in Pittsburgh, Pennsylvania, USA. She also serves as an Assistant Professor of Medicine, Clinical and Translational Science, and Nursing at the University of Pittsburgh. Dr. Broyles is the recipient of a 5-year VA Career Development Award from the Health Services Research \& Development service of the VA.

MEW is a Research Health Science Specialist at the VAPHS CHERP. Dr. Wieland serves as Study Coordinator for this trial.

ALC is a Research Health Science Specialist at CHERP. She serves as the Senior Research Assistant for this trial.

MMD is a nurse practitioner in the Endocrinology Outpatient Clinic at VAPHS. She serves as the nurse interventionist on this study.

KLK is a Professor of Medicine, Clinical \& Translational Science, and Health Policy \& Management at the University of Pittsburgh and Director of the University of Pittsburgh General Internal Medicine Fellowship Program. $\mathrm{BHH}$ is a statistician with MIRECC

AOY is a senior statistician/affiliate investigator with CHERP. Dr. Youk serves as the senior statistician for this trial. She is an Assistant Professor of Biostatistics, Epidemiology and Clinical \& Translational Science in the Graduate School of Public Health at the University of Pittsburgh. AJG is a CHERP Core Investigator, Clinical Director of the VISN 4 MIRECC, and a staff physician at VAPHS. He also serves as an Associate Professor and Advisory Dean at the University of Pittsburgh School of Medicine. MAS is a Professor of Medicine in the Department of Population Health at the New York University School of Medicine, New York, New York, USA.

\section{Acknowledgements}

We acknowledge the staff nurses from the 4 West, 5 West, and 6 West patient care units, and the leadership at VAPHS for their support of the study. Funding for this study was provided by the Nursing Research Initiative 
of the Health Services Research and Development (HSR \& D) Service of the VA (1 IO1 HX000802-01, PI: Lauren M. Broyles). Dr. Broyles is currently supported by a Career Development Award (CDA 10-014) from the HSR \& D Service of the $V A$. The material is the result of work supported with resources and the use of facilities at VAPHS. The views expressed in this article are those of the authors and do not necessarily reflect the position or policy of the VA or the United States Government.

\section{Author details}

${ }^{1}$ Center for Health Equity Research and Promotion, Veterans Affairs (VA) Pittsburgh Healthcare System, University Drive C (151C), Pittsburgh, PA 15240, USA. ${ }^{2}$ Division of General Internal Medicine, Department of Medicine, School of Medicine, University of Pittsburgh, 230 McKee Place, Suite 600, Pittsburgh, PA 15213, USA. ${ }^{3}$ Veterans Integrated Service Network 4 (VISN4) Mental Illness Research, Education, and Clinical Center, VA Pittsburgh Healthcare System, University Drive C (151C), Pittsburgh, PA 15240, USA ${ }^{4}$ Department of Biostatistics, Graduate School of Public Health, University of Pittsburgh, Pittsburgh, PA, USA. ${ }^{5}$ Department of Population Health, Center for Healthful Behavior Change, New York University School of Medicine, 227 East 30th Street, New York, NY 10016, USA.

\section{Received: 31 December 2014 Accepted: 22 April 2015} Published online: 13 May 2015

\section{References}

1. U.S. Preventive Services Task Force. Final recommendation statement: alcohol misuse: screening and behavioral counseling interventions in primary care. May 2013. [http://www.uspreventiveservicestaskforce.org/ Page/Topic/recommendation-summary/alcohol-misuse-screening-andbehavioral-counseling-interventions-in-primary-care]

2. National Institute on Alcohol Abuse and Alcoholism. Helping patients who drink too much: a clinician's guide. National Institute on Alcohol Abuse and Alcoholism. Updated 2005 edition. [http://pubs.niaaa.nih.gov/publications/ Practitioner/CliniciansGuide2005/clinicians_guide.htm]

3. Saitz R. Clinical practice. Unhealthy alcohol use. N Engl J Med. 2005;352:596-607.

4. The Joint Commission. Specifications manual for national hospital inpatient quality measures. [http://www.jointcommission.org/specifications_manual_for_ national_hospital_inpatient_quality_measures]

5. The Joint Commission. Substance use. October 23, 2014. [http:// www.jointcommission.org/substance_use]

6. National Quality Forum. NQF endorses behavioral health measures. March 7 2014. [http://www.qualityforum.org/News_And_Resources/Press_Releases/ 2014/NQF_Endorses_Behavioral_Health_Measures.aspx]

7. Saitz R. Candidate performance measures for screening for, assessing, and treating unhealthy substance use in hospitals: advocacy or evidence-based practice? Ann Intern Med. 2010;153:40-3.

8. Saitz R. Candidate performance measures for screening for, assessing, and treating unhealthy substance use in hospitals. Ann Intern Med. 2011;154:73-4.

9. Madras BK. Candidate performance measures for screening for, assessing, and treating unhealthy substance use in hospitals. Ann Intern Med. 2011:154:72-3. doi:10.7326/0003-4819-154-1-201101040-00016.

10. Loeb JM, Watt AE, Lawler NK. Candidate performance measures for screening for, assessing, and treating unhealthy substance use in hospitals. Ann Intern Med. 2011;154:72. doi:10.7326/0003-4819-154-1-201101040-00015.

11. Gentilello LM, Goplerud E. Candidate performance measures for screening for, assessing, and treating unhealthy substance use in hospitals. Ann Intern Med. 2011;154:73. doi:10.7326/0003-4819-154-1-201101040-00017.

12. Saitz R, Palfai TP, Cheng DM, Horton NJ, Freedner N, Dukes K, et al. Brief intervention for medical inpatients with unhealthy alcohol use: a randomized, controlled trial. Ann Intern Med. 2007;146:167-76.

13. Saitz R, Palfai TP, Cheng DM, Horton NJ, Dukes K, Kraemer KL, et al. Some medical inpatients with unhealthy alcohol use may benefit from brief intervention. J Stud Alcohol Drugs. 2009;70:426-35.

14. Freyer-Adam J, Coder B, Baumeister SE, Bischof G, Riedel J, Paatsch K, et al. Brief alcohol intervention for general hospital inpatients: a randomized controlled trial. Drug Alcohol Depend. 2008;93:233-43.

15. Mcmanus S, Hipkins J, Haddad P, Guthrie E, Creed F. Implementing an effective intervention for problem drinkers on medical wards. Gen Hosp Psychiatry. 2003;25:332-7.
16. Holloway AS, Watson HE, Arthur AJ, Starr G, McFadyen AK, Mclntosh J. The effect of brief interventions on alcohol consumption among heavy drinkers in a general hospital setting. Addiction. 2007;102:1762-70.

17. Williams EC, Palfai T, Cheng DM, Samet JH, Bradley KA, Koepsell TD, et al. Physical health and drinking among medical inpatients with unhealthy alcohol use: a prospective study. Alcohol Clin Exp Res. 2010;34:1257-65.

18. Tsai MC, Tsai YF, Hwang FM, Liu CY. Effectiveness of a brief intervention for managing hazardous drinking problems of inpatients in Taiwan. J Adv Nurs. 2011;67:2038-46

19. Tsai YF, Tsai MC, Lin YP, Chen CY. Brief intervention for problem drinkers in a Chinese population: a randomized controlled trial in a hospital setting. Alcohol Clin Exp Res. 2009;33:95-101.

20. Liu SI, Wu SI, Chen SC, Huang HC, Sun FJ, Fang CK, et al. Randomized controlled trial of a brief intervention for unhealthy alcohol use in hospitalized Taiwanese men. Addiction. 2011;106:928-40.

21. Bernstein JA, Bernstein E, Heeren TC. Mechanisms of change in control group drinking in clinical trials of brief alcohol intervention: implications for bias toward the null. Drug Alcohol Rev. 2010;29:498-507.

22. Heather N. Toward an understanding of the effective mechanisms of alcohol brief interventions. Alcohol Clin Exp Res. 2014;38:626-8.

23. Kypri K. Methodological issues in alcohol screening and brief intervention research. Subst Abus. 2007;28:31-42.

24. Moreira MT, Oskrochi R, Foxcroft DR. Personalised normative feedback for preventing alcohol misuse in university students: solomon three-group randomised controlled trial. PLoS One. 2012;7:e44120.

25. Clifford PR, Maisto SA. Subject reactivity effects and alcohol treatment outcome research. J Stud Alcohol. 2000;61:787-93.

26. Donovan DM, Bogenschutz MP, Perl H, Forcehimes A, Adinoff B, Mandler R, et al. Study design to examine the potential role of assessment reactivity in the screening, motivational assessment, referral, and treatment in emergency departments (SMART-ED) protocol. Addict Sci Clin Pract. 2012;7:16.

27. Katzman R, Brown T, Fuld P, Peck A, Schechter R, Schimmel $H$. Validation of a short orientation-memory-concentration test of cognitive impairment. Am J Psychiatry. 1983;140:734-9.

28. U.S. Department of Veterans Affairs. Substance use disorders (SUD) VA/DoD clinical practice guidelines. [http://www.healthquality.va.gov/guidelines/MH/ sud/sud_full_601f.pdf]

29. Bush K, Kivlahan DR, McDonell MB, Finn SD, Bradley KA. The AUDIT alcohol consumption questions (AUDIT-C): an effective brief screening test for problem drinking. Ambulatory care quality improvement project (ACQUIP). Alcohol use disorders identification test. Arch Intern Med. 1998;158:1789-95.

30. Bradley KA, Bush KR, Epler AJ, Dobie DJ, Davis TM, Sporleder JL, et al. Two brief alcohol-screening tests from the alcohol use disorders identification test (AUDIT): validation in a female Veterans affairs patient population. Arch Intern Med. 2003:163:821-9.

31. Cottler LB, Robins LN, Helzer JE. The reliability of the CIDI-SAM: a comprehensive substance abuse interview. Br J Addict. 1989:84:801-14.

32. American Psychological Association. Diagnostic and statistical manual of mental disorders. 4th ed. Washington, DC: American Psychological Association; 2000.

33. Centers for Disease Control and Prevention. Behavioral risk factor surveillance system survey questionnaires. [http://www.cdc.gov/brfss/questionnaires.htm]

34. D'Onofrio G, Bernstein E, Bernstein J, Woolard RH, Brewer PA, Craig SA, et al. Patients with alcohol problems in the emergency department, part 2: intervention and referral. SAEM substance abuse task force. Society for academic emergency medicine. Acad Emerg Med. 1998;5:1210-7.

35. U.S. Department of Veterans Affairs, Quality Enhancement Research. Brief alcohol counseling for alcohol misuse. [http://www.queri.research.va.gov/ tools/alcohol-misuse/alcohol-counseling.cfm]

36. U.S. Department of Veterans Affairs, Office of Public Health and Environmental Standards, Hepatitis C Program. Reducing alcohol use with brief intervention: toolkit resources. [http://www.hepatitis.va.gov/products/ brief-intervention-resources.asp]

37. National Institutes of Health. Rethinking drinking: alcohol and your health. National Institute on Alcohol Abuse and Alcoholism. 2010. [http:// rethinkingdrinking.niaaa.nih.gov/]

38. Babor TF, Higgins-Biddle JC. Brief intervention for hazardous and harmful drinking: a manual for use in primary care. Geneva: World Health Organization; 2001

39. Center for Substance Abuse Treatment. Brief interventions and brief therapies for substance abuse; treatment improvement protocol (TIP) series 
34. Rockville: Substance Abuse and Mental Health Services Administration; 1999. HHS Publication Number: (SMA) 12-3952.

40. Soderstrom CA, DiClemente CC, Dischinger PC, Hebel JR, McDuff DR, Auman KM, et al. A controlled trial of brief intervention versus brief advice for at-risk drinking trauma center patients. J Trauma. 2007;62:1102-11.

41. Lindquist R, Wyman JF, Talley KM, Findorff MJ, Gross CR. Design of control-group conditions in clinical trials of behavioral interventions. J Nurs Scholarsh. 2007:39:214-21.

42. Miller WR, Tonigan JS. Assessing drinkers' motivation for change: the stages of change readiness and treatment eagerness scale (SOCRATES). Psychol Addict Behav. 1996;10:81-9.

43. Miller WR, Tonigan JS, Longabaugh R. The drinker inventory of consequences (DrlnC). An instrument for assessing adverse consequences of alcohol abuse. Test manual. National Institute on Alcohol Abuse and Alcoholism Project MATCH Monograph Series, Vol. 4; 1995. U.S. Department of Health and Human Services, Public Health Service, National Institutes of Health. NIH Publication no. 95-3911.

44. Dane AV, Schneider BH. Program integrity in primary and early secondary prevention: are implementation effects out of control? Clin Psychol Rev. 1998;18:23-45

45. Miller WR, Rollnick S. Motivational interviewing: preparing people for change. 2nd ed. New York, NY: Guilford Press; 2002.

46. Prochaska JO, DiClemente CC. Stages and processes of self-change of smoking: toward an integrative model of change. J Consult Clin Psychol. 1983:51:390-5.

47. Prochaska JO, DiClemente CC, Norcross JC. In search of how people change. Applications to addictive behaviors. Am Psychol. 1992;47:1102-14

48. Motivational Interviewing Network of Trainers. About MINT: motivational interviewing network of trainers. [http://motivationalinterviewing.org/]

49. Broyles LM, Rodriguez KL, Kraemer KL, Sevick MA, Price PA, Gordon AJ. A qualitative study of anticipated barriers and facilitators to the implementation of nurse-delivered alcohol screening, brief intervention, and referral to treatment for hospitalized patients in a Veterans Affairs medical center. Addict Sci Clin Pract. 2012;7:7.

50. O'Connor PG. Brief interventions for problem drinking: another piece of the puzzle. Ann Intern Med. 2007;146:223-5.

51. Saitz R. Alcohol screening and brief intervention in primary care: absence of evidence for efficacy in people with dependence or very heavy drinking. Drug Alcohol Rev. 2010;29:631-40

52. Moyer A, Finney JW, Swearingen CE, Vergun P. Brief interventions for alcohol problems: a meta-analytic review of controlled investigations in treatment-seeking and non-treatment-seeking populations. Addiction. 2002;97:279-92.

53. Jonas DE, Garbutt JC, Amick HR, Brown JM, Brownley KA, Council CL, et al. Behavioral counseling after screening for alcohol misuse in primary care: a systematic review and meta-analysis for the U.S. preventive services task force. Ann Intern Med. 2012;157:645-54.

54. Delaney KE, Lee AK, Lapham GT, Rubinsky AD, Chavez LJ, Bradley KA. Inconsistencies between alcohol screening results based on AUDIT-C scores and reported drinking on the AUDIT-C questions: prevalence in two US national samples. Addict Sci Clin Pract. 2014:9:2.

\section{Submit your next manuscript to BioMed Central and take full advantage of:}

- Convenient online submission

- Thorough peer review

- No space constraints or color figure charges

- Immediate publication on acceptance

- Inclusion in PubMed, CAS, Scopus and Google Scholar

- Research which is freely available for redistribution

Submit your manuscript at www.biomedcentral.com/submit 